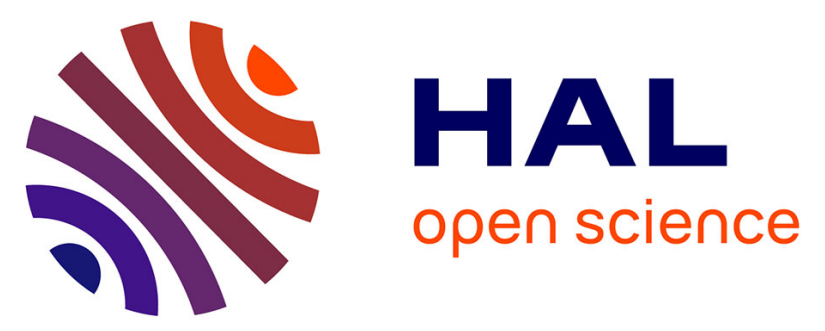

\title{
Les effets de la livraison à domicile sur l'accès aux produits alimentaires: le cas des grandes surfaces alimentaires et des cybermarchés de l'aire urbaine dijonnaise
}

Benjamin Motte-Baumvol, Leslie Belton-Chevallier, Myriam Schoelzer, Guillaume Carrouet

\section{To cite this version:}

Benjamin Motte-Baumvol, Leslie Belton-Chevallier, Myriam Schoelzer, Guillaume Carrouet. Les effets de la livraison à domicile sur l'accès aux produits alimentaires: le cas des grandes surfaces alimentaires et des cybermarchés de l'aire urbaine dijonnaise. Flux - Cahiers scientifiques internationaux Réseaux et territoires, 2012, 2012 (88), 19p. hal-00931709

\author{
HAL Id: hal-00931709 \\ https://hal.science/hal-00931709
}

Submitted on 27 Feb 2014

HAL is a multi-disciplinary open access archive for the deposit and dissemination of scientific research documents, whether they are published or not. The documents may come from teaching and research institutions in France or abroad, or from public or private research centers.
L'archive ouverte pluridisciplinaire HAL, est destinée au dépôt et à la diffusion de documents scientifiques de niveau recherche, publiés ou non, émanant des établissements d'enseignement et de recherche français ou étrangers, des laboratoires publics ou privés.

$$
\text { Copyright }
$$


Les effets de la livraison à domicile sur l'accès aux produits alimentaires : le cas des grandes surfaces alimentaires et des cybermarchés de l'aire urbaine dijonnaise

\author{
Auteurs : \\ Benjamin Motte-Baumvol $^{1}$ \\ Leslie Belton-Chevallier ${ }^{2}$ \\ Miriam Schoelzel ${ }^{1}$ \\ Guillaume Carrouet $^{1}$
}

Août 2012

\footnotetext{
${ }^{1}$ Université de Bourgogne, ThéMA (UMR 6049), 2 Boulevard Gabriel, 21000 DIJON

${ }^{2}$ IFSTTAR, DEST, 2 rue de la Butte Verte, 93166 NOISY-LE-GRAND CEDEX
} 
Les effets de la livraison à domicile sur l'accès aux produits alimentaires : le cas des grandes surfaces alimentaires et des cybermarchés de l'aire urbaine dijonnaise

Mots clés : livraison à domicile, supermarché, cybermarché, grande surface alimentaire, accessibilité, périurbain, Dijon

Résumé : L'accessibilité des populations aux Grandes Surfaces Alimentaires (GSA) est différenciée selon les espaces. Si une très grande majorité de périurbains n'éprouvent pas de difficultés pour y accéder, certains y font face plus difficilement. Pour ces ménages en particulier, la livraison à domicile, constitue un moyen d'améliorer leur accès en supprimant les difficultés liées aux déplacements. A partir d'une recension de l'offre et d'une enquête par entretiens auprès des principaux acteurs de la livraison à domicile de l'aire urbaine dijonnaise, nos résultats mettent en évidence une offre de livraison à domicile peu développée et encore balbutiante. Spatialement concentrée au centre de l'aire urbaine, les aires de livraison tendent à favoriser un peu plus encore les populations urbaines plus proches des GSA au détriment des populations périurbaines périphériques.

The home-delivery effects on grocery accessibility: the case study of supermarkets, hypermarkets and cybermarkets in Dijon's area (France).

Keywords : home delivery, supermarket, cybermarket, e-grocery, accessibility, suburbs, Dijon

Abstract : The population's access to groceries varies with the kind of spaces people live in (center vs periphery). If a large part of peri-urban peoples do not experience difficulties in accessing to supermarkets, some of them will face more difficulties. For these households, home delivery may reduce disparities of access between spaces and improve access to a wider assortment of groceries. The empirical work of this study is mainly based on the census of offers from several statistical sources, and a survey by interviews with local actors of home delivery. Our results show that home delivery supply is spatially concentrated. The delivery areas tend to promote a little more the populations of the center already close to a supermarket. Moreover, according to their schedules and their fares, some delivery services target a small sample of customers in order not to cause an explosion of their distribution costs. 


\section{Introduction}

L'accessibilité des populations aux Grandes Surfaces Alimentaires ${ }^{3}$ (GSA) est différenciée selon les espaces. Alors qu'au cœur des villes, les urbains peuvent accéder à pieds à une offre diversifiée, les périurbains et les ruraux doivent parcourir plusieurs kilomètres en voiture pour accéder au supermarché le plus proche (Motte-Baumvol 2008). Si une très grande majorité de périurbains n'éprouvent pas de difficultés, certains y font face plus difficilement tels que les ménages sans voiture, les personnes âgées ou les actifs avec des horaires de travail étendus. Pour ces ménages, la livraison à domicile, notamment à partir de la vente en ligne, peut constituer un moyen d'améliorer leur accès à un assortiment de produits alimentaires en supprimant les difficultés liées aux déplacements. Dans ce contexte, la livraison à domicile est un mode de distribution en mesure de bouleverser les cadres d'analyse de l'accessibilité des populations au commerce, plus particulièrement dans les espaces périurbains.

La livraison à domicile est un service proposé par les GSA soit à travers l'achat en ligne, soit après l'achat en magasin qui consiste à acheminer au domicile du client les marchandises que ce dernier a acheté. Aujourd'hui, le chiffre d'affaires généré par ces services est difficile à estimer. A l'échelle nationale, l'achat en ligne dans les cybermarchés reste une pratique peu courante. Par exemple, le chiffre d'affaires du cybermarché AuchanDirect correspondrait ainsi à $45 \%$ de celui d'un seul hypermarché de l'enseigne ${ }^{4}$. Les autres cybermarchés auraient des chiffres d'affaires encore inférieurs. De plus, avec le développement des systèmes de retraits intermédiaires (drive ou guichet en magasin), achat de produits alimentaires en ligne et livraison à domicile ne vont plus forcément de pair. Pour la livraison à domicile à partir d'achats en magasin, peu de données sont disponibles. $\mathrm{A}$ I'heure actuelle, en France, la plupart des clients se rendent plutôt en magasin pour effectuer leurs achats alimentaires et ramènent eux-mêmes leurs achats à domicile (Ranvier et Sury 2009). La livraison à domicile de produits d'épicerie à partir de la vente en ligne ou de la vente en magasin ne s'adresse a priori qu'à une minorité de ménages et d'individus. Murphy (2007) voit six segments de clientèle qui ont recours à la livraison à domicile à partir de la vente en ligne, segments qui peuvent également concerner la livraison à partir de la vente en magasin :

- Les ménages sans voiture (par choix ou par nécessité) pour lesquels le transport des courses hebdomadaires pose problème.

- Les personnes à mobilité réduite ou âgées pour qui les courses peuvent être épuisantes et fatigantes.

\footnotetext{
${ }^{3}$ Par définition, les Grandes Surfaces Alimentaires (INSEE 2010) regroupent l'ensemble des établissements de commerce de détail non spécialisés ou généralistes dont la superficie est supérieure à $400 \mathrm{~m}^{2}$ et qui réalisent une partie de leur chiffre d'affaire en produits alimentaires. Aussi appelées Grandes et Moyennes Surfaces, les GSA regroupent les supermarchés et les hypermarchés. Les supermarchés ont une superficie comprise en 400 et $2.500 \mathrm{~m}^{2}$ et réalisent au moins $2 / 3$ de leur chiffre d'affaire en alimentation. Les hypermarchés font entre 2.500 et $15.000 \mathrm{~m}^{2}$. Leur chiffre d'affaire est généré pour un tiers par des produits alimentaires. Les GSA se caractérisent donc par un assortiment d'abord alimentaire mais proposent aussi un éventail plus ou moins large de produits non alimentaires.

${ }^{4}$ Compte sociaux Auchandirect 2008
} 
- Les ménages avec des enfants en bas âge pour lesquels les courses demandent une organisation et une logistique importante.

- Les clients qui cherchent des produits spécifiques ou biologiques que l'on ne trouve pas dans tous les supermarchés.

- Les personnes qui travaillent beaucoup ou ont une vie sociale très active. Ils n'ont donc que peu de temps libre pour aller faire leurs courses et leurs horaires coïncident difficilement avec ceux des magasins.

- Les technophiles qui préfèrent l'utilisation d'un appareil électronique à la socialisation d'une grande surface.

Pour la livraison à domicile associée à la vente en magasin, les trois premiers segments de clientèle paraissent également susceptibles d'utiliser ce service car ils peuvent difficilement effectuer le transport et la manutention des achats. Pour les trois derniers types de clientèles, le transport et la manutention des achats ne paraissent pas être une question clé. Par un manque de disponibilité temporelle (liée à leur niveau d'activité) ou des produits recherchés, la vente en ligne tendrait plutôt à se substituer, totalement ou en partie, à des formes de vente traditionnelles en magasin. En se basant sur cette typologie, la demande de service de livraison à domicile serait susceptible de s'accroître dans les années à venir. Plusieurs évolutions socioéconomiques le laissent présager: développement des usages d'Internet et adoption croissante de la vente en ligne, vieillissement de la population, étalement urbain, maintien d'un taux de fécondité important, raréfaction des ressources fossiles, etc.

Dans un contexte d'accroissement du coût des déplacements liés à l'énergie et d'une volonté de développement durable, la livraison à domicile constituerait un moyen pour diminuer le nombre des déplacements et les volumes de gaz émis en améliorant la desserte de territoires peu denses et en réduisant leur niveau de dépendance automobile. D'un point de vue environnemental, les impacts de la livraison à domicile en tant que mode de livraison de la vente à distance sont discutés (Cullinane 2009, Cairns 2005). Par contre, la question de l'équité sociale de desserte des territoires est peu débattue. Internet a souvent été considéré comme un moyen d'abolir les distances (Cairncross 1997) et de favoriser l'étalement urbain (Shen 2000). Appliquer ce raisonnement à l'achat en ligne de biens matériels en général et de produits alimentaires en particulier reviendrait à oublier que le processus d'achat est électronisable en partie et qu'il peut donc avoir des effets potentiellement multiples sur l'organisation spatiale du commerce, du renforcement de zones périphérique ou centrales à l'émergence d'un maillage plus fin des territoires (Rallet 2001). En déléguant aux distributeurs ces étapes du processus d'achat (préparation de la commande et/ou livraison), l'accessibilité aux produits alimentaires des territoires, notamment les moins denses, tend-t-elle à s'accroître ? Certains distributeurs n'hésitent pas à l'affirmer : " faire ses courses en ligne, que l'on habite une grande ville ou une zone rurale isolée, c'est désormais possible grâce à Placedumarché.fr, le nouveau site marchand qui livre à domicile" (site de l'enseigne en 2011). Cela suppose que les distributeurs desservent de manière égalitaire tous types de territoires. Pour le vérifier, il est alors nécessaire de se rendre compte des périmètres effectivement desservis par les enseignes qui proposent la livraison à domicile.

Nous nous baserons sur l'étude d'un territoire spécifique, à savoir l'aire urbaine dijonnaise. A partir d'une recension de l'offre et d'une enquête par entretiens auprès des principaux acteurs de la livraison à domicile pour les GSA et les cybermarchés, leurs équivalents en ligne (Ranvier et Sury 
2009), nos résultats mettent en évidence une offre de livraison à domicile peu développée et encore balbutiante. Spatialement concentrée au centre de l'aire urbaine, les aires de livraison tendent à favoriser un peu plus encore les populations urbaines plus proches des GSA au détriment des populations périurbaines périphériques. Par ailleurs, par des horaires limités, des tarifs peu attractifs et l'absence de publicité, certains services de livraisons ciblent d'emblée une clientèle réduite afin de ne pas entraîner une explosion des coûts de distribution pour les distributeurs qui les pratiquent. A l'inverse, d'autres enseignes voudraient viser des aires de chalandise et une clientèle plus larges. Mais, elles sont probablement handicapées par des coûts logistiques et de fonctionnement importants, faute d'investissements nécessaires, trop conséquents pour la taille du marché dijonnais.

\section{Définitions et méthode}

\section{GSA et cybermarchés}

Les GSA offrent un assortiment de biens alimentaires comportant trois grands types de produits : de l'épicerie, des produits frais et des surgelés. Dans ce travail, nous nous intéresserons uniquement aux distributeurs qui offrent l'ensemble de ces trois types de produits. Ainsi, les offres relevant de I'alimentation spécialisée qui proposent seulement des surgelés, des paniers de fruits et légumes ou des produits d'épicerie sèche ne sont pas prises en compte. En effet, elles ne correspondent pas au mode d'approvisionnement habituel des ménages qui, dans leur écrasante majorité, utilisent les GSA comme leur source principale d'approvisionnement alimentaire. En 2010, les GSA ont réalisé $66,6 \%$ des parts de marchés du commerce de détail de produits alimentaires (Bras et al. 2012). Par ailleurs, ces distributeurs spécialisés nécessiteraient un surcroît d'effort pour l'approvisionnement de la plupart des ménages en multipliant les sources et les modes de distribution.

Sur Internet, les distributeurs de produits alimentaires qui offrent de façon combinée de l'épicerie, du frais et des surgelés sont faciles à identifier. On les désigne couramment sous le vocable de cybermarché ou de "e-grocer » dans la littérature anglophone. En France, ils appartiennent pour la plupart aux grandes enseignes du commerce intégré qui disposent de magasins physiques. Ces cybermarchés sont donc des "click and mortar » ou "bricks and clicks " dans la mesure où le ecommerce est un canal supplémentaire, qui vient compléter les points de vente physiques dont l'enseigne dispose (références). Dans le secteur des cybermarchés en France, depuis le rachat de Télémarket par Super U, il n'y a plus de " pure-players ", c'est-à-dire de distributeur dont Internet est le seul canal. Premier entrant dans le secteur des cybermarchés et à l'époque pure player, Télémarket est aujourd'hui au service de la stratégie multicanal de Super $U$ et est donc devenu un click-and-mortar (même si la distribution en magasins n'était pas son métier d'origine) comme beaucoup de pure-players de l'épicerie électronique dans le monde (Durand et Senkel 2007). Si ces enseignes distinguent ou ont parfois distingué leurs cybermarchés de leurs magasins physiques en utilisant des appellations différentes ${ }^{5}$, leur appartenance aux enseignes est clairement affichée sur

\footnotetext{
${ }^{5}$ Créé en 1999, le site marchand de Carrefour a d'abord été appelé Ooshop avant de repasser sous appellation Carrefour en 2009. Lancé en 1983 sur minitel puis en 1998 sur Internet par le groupe Galeries Lafayette, Télémarket est devenue une filiale de Super U en 2011 et a été rebaptisé Utélémarket. Le site marchand de Cora inauguré en 2000 s'appelle toujours Houra.fr mais le nom de l'enseigne mère est visible d'emblée sur le site. Les enseignes qui se sont lancées plus récemment dans l'épicerie en ligne comme Auchan (2001), Intermarché (2004), Casino (qui a ouvert un $2^{\mathrm{e}}$ site marchant en nom propre en 2009) ou Monoprix (dont le site
} 
les pages web de leur site marchand. Cette visibilité du distributeur offre un avantage en termes d'image : elle garantie au client un certain niveau de service, un assortiment avec un nombre et une diversité de références analogue à l'offre en magasin. De ce point de vue, l'offre des cybermarchés affiche une grande homogénéité et correspond à celle d'une grande surface alimentaire compte tenu de la taille de leur assortiment, comprise entre 6.500 et 50.000 références (Durand 2008 ; Dumans et Chambolle 2002).

\section{La collecte des données sur la livraison à domicile dans l'aire urbaine et sur l'aire urbaine}

Tout d'abord, pour les cybermarchés, leurs sites Internet ont servi de sources principales d'informations en renseignant sur les offres dans l'aire urbaine dijonnaise, leurs périmètres, les horaires ou les coûts de la livraison. Pour l'offre de magasins et de livraison à domicile à partir de l'achat en magasin, nous nous sommes appuyés sur plusieurs sources et méthodes pour collecter les données. Dans un premier temps, nous avons eu recours à l'« Atlas de la distribution 2010 ", publié annuellement par le magazine LSA (Libre Service Actualités), revue professionnelle spécialisée dans la grande distribution et la consommation. L'Atlas de la distribution a permis de déterminer l'enseigne, la taille ou les coordonnées de chaque magasin. Toutefois, il n'indique pas les services proposés en magasin comme la livraison à domicile. Les sites Internet des différentes enseignes ont servi à recueillir des informations détaillées sur la livraison à domicile à partir de la vente en magasin, notamment les horaires, les frais et les zones de livraison. En 2011, les informations des deux précédentes sources ont été vérifiées et complétées par des visites et des appels téléphoniques aux différents distributeurs de l'aire urbaine. Le service de livraison à domicile à partir de la vente en magasin est généralement peu décrite sur les sites web des enseignes d'où la nécessité de vérifier directement dans les magasins concernés. Lors de cette étape, plusieurs informations obtenues précédemment ont aussi été actualisées. Un supermarché s'est révélé fermé alors qu'il figurait encore sur le site web de l'enseigne et dans l'Atlas de la distribution. Sur sa page web, une autre grande surface déclarait offrir la livraison dans la liste des services disponibles en magasin alors que ce service n'y était plus disponible. Enfin, des déplacements dans plusieurs supermarchés ont été l'occasion d'observer comment les magasins communiquent autour de la livraison à domicile et l'intègrent dans leur dispositif de vente avec, par exemple, l'existence d'une caisse ou d'un accueil réservé.

\section{Les entretiens}

La deuxième étape du travail de terrain a consisté à réaliser des entretiens pour compléter les connaissances disponibles sur la livraison effectuée par les grandes surfaces de l'aire urbaine dijonnaise. Ces entretiens semi-directifs auprès des responsables de magasins et de prestataires locaux de livraison cherchent à éclairer le fonctionnement et l'organisation de la livraison à domicile. II s'agit de comprendre depuis quand et pour quelles raisons la livraison est proposée, quel est le segment de clients ciblé et quels sont les clients qui utilisent avant tout la livraison. Pour les enseignes où ce n'était pas encore clair, ces entretiens ont aussi permis de connaître leur zone de livraison et les critères qui ont été retenus pour sa définition.

propre a été lancé en 2008) ont de suite opté pour garder une référence claire à l'enseigne dans leur nom de site. 
Les premiers entretiens ont été réalisé auprès du magasin Géant Casino de Fontaine-Lès-Dijon et son prestataire Maxi'M Service. Le deuxième magasin qui a été choisi pour un entretien était le Super U de Chenôve. Ce magasin a proposé la livraison à domicile pendant un certain temps mais ne le fait plus aujourd'hui. Un entretien avec la responsable de la livraison du Monoprix de Dijon a également été effectué. Ce magasin a été choisi car il se trouve en centre-ville, il propose de la vente en ligne et en magasin et qu'il livre au-delà de Dijon intra-muros, sur une large partie de l'aire urbaine. Enfin, un entretien a été effectué avec le magasin Intermarché Port du Canal qui centralise l'ensemble des livraisons des magasins Intermarché localisés à et autour de Dijon (sur 7 magasins). Un entretien a notamment été réalisé avec le prestataire des livraisons du Super $U$ de Talant. Ce prestataire autoentrepreneur dispose d'un accord verbal du magasin qui lui permet d'offrir la livraison aux clients du supermarché. La livraison n'est donc ni organisée, ni proposée par le personnel du supermarché.

\section{RESULTATS}

\section{Une offre de livraison à domicile réduite dans l'aire urbaine dijonnaise}

En mai 2011, l'aire urbaine dijonnaise enregistre une soixantaine de grandes surfaces alimentaires. Un quart de ces grandes surfaces sont situées dans Dijon. Une moitié est localisée dans les autres communes du pôle urbain, en particulier dans les communes de Chenôve, Talant, Fontaine-Lès-Dijon et Quetigny. Ces communes sont contiguës à Dijon et/ou situées le long des trois principaux corridors routiers radiaux de l'agglomération (au sud, à l'est et au nord). Les autres grandes surfaces sont localisées dans la plaine de la Saône (au sud-est de Dijon), là où les densités de populations y sont les plus fortes, autour des communes de Genlis et Brazey-en-Plaine. A l'ouest et au nord, l'équipement en grandes surfaces alimentaires est presque inexistant. De fait, ces magasins sont fortement concentrés spatialement: sur les 214 communes de l'aire urbaine, seules 21 disposent d'un supermarché soit $10 \%$ d'entre elles. Cette concentration est à l'image de la population puisque $74 \%$ de la population et $79 \%$ des ménages disposent d'un supermarché dans leur commune de résidence.

Les 60 magasins de l'aire urbaine dijonnaise appartiennent à 15 enseignes différentes dont près d'un tiers de hard discount qui offrent des niveaux de prix et de services moins élevés. Si de nombreuses enseignes sont présentes sur le secteur géographique étudié, l'offre de produits alimentaires en ligne reste limitée. Alors que toutes les principales enseignes de la grande distribution française se sont dotées d'un site de vente en ligne, seules trois d'entre elles sont présentes en livrant dans l'aire urbaine de Dijon. Aucun des principaux cybermarchés, tels que Carrefour (anciennement Ooshop), UTélémarket (Super U), Houra.fr (Cora) ou Auchandirect (Auchan) ne proposent localement ce service. Ils laissent ainsi la place à des acteurs secondaires de la vente en ligne: Géant Casino, Intermarché et Monoprix. Cette situation est la conséquence directe des différentes organisations logistiques des cybermarchés qui reposent soit sur la préparation en entrepôts dédiés (un entrepôt unique avec plateformes de dégroupage ou des entrepôts locaux de préparation de commandes), soit sur la préparation de commandes en magasins (Gavaud 2010). Les principales enseignes comme Carrefour, UTélémarket ou Auchandirect privilégient une organisation logistique dédiée, autour d'entrepôts automatisés à partir desquels s'opère la distribution. Ce modèle d'organisation dit du "in-warehouse picking" a pour avantage un coût réduit de logistique et de préparation des commandes. Mais, elle nécessite un investissement important et ne se justifie que si l'activité dépasse plusieurs centaines de commandes par jour (Koster 2002). A l'inverse, pour les acteurs secondaires, le modèle de préparation des commandes par prélèvements dans un magasin existant 
(ou "in-store picking ") s'est imposée sur la base du modèle initié par Tesco, acteur majeur de la grande distribution britannique (Murphy 2007). Ce mode d'organisation nécessite un investissement réduit, correspond à un chiffre d'affaires relativement faible et se déploie rapidement à partir du réseau de magasins existants : il s'adapte donc mieux à des marchés et des distributeurs de petites tailles (Brousseau et Kessous 2003) comme le marché dijonnais. En contrepartie, le coût unitaire d'une commande est plus important car l'organisation implique qu'un préparateur va prélever les produits dans les rayons. Ce va-et-vient peut perturber les clients en train de faire leurs courses en magasin et entraîner leur fuite (Ogawara et al 2003) et s'avérer coûteux dans des grands magasins présentant un grand nombre de références (Brousseau et Kessous 2003).

Plus que la préparation des commandes, la livraison à domicile coûte cher aux cybermarchés. En effet, ce ne sont plus les clients qui ont la charge du transport final, du « dernier kilomètre " mais les vendeurs (Rallet 2001 ; Li et Yousept 2004 ; Augereau et al. 2009). Or, ce coût ne peut être reporté entièrement sur le consommateur: ce dernier n'est pas prêt à payer des frais de livraison conséquents pour des produits à faible valeur comme les produits alimentaires (Rallet 2001). A partir du moment où le vendeur prend en charge les coûts de transport, il va chercher à les minimiser. Or, la livraison à domicile impose le transport de petites quantités, avec un camion tri-température et une forte dispersion des destinations. De plus, la livraison à domicile est marquée par de nombreux aléas, tels la congestion, les difficultés de stationnement ou d'accès à certains logements ainsi que les absences des clients (Gratadour 2001) qui renchérissent d'autant le coût de la livraison. C'est pourquoi les cybermarchés optent de plus en plus pour d'autres formules logistiques, à savoir le retrait de courses opérées en ligne sur rendez-vous et directement dans les magasins de l'enseigne ou dans un "drive ", c'est-à-dire dans un entrepôt de proximité situé en zone commerciale (Gavaud 2010). Pour la vente en ligne, le retrait des achats en magasin représenterait une économie de $70 \%$ pour le vendeur par rapport à la livraison à domicile (Durand 2010).

Les coûts inhérents à la livraison à domicile sont aussi présents pour les grandes surfaces qui proposent de livrer les courses opérées par les clients dans leurs rayons. Si les coûts de préparation sont moindres (limités à la manutention des produits entre la sortie de caisse et le point d'accueil pour la livraison), les coûts du transport des marchandises achetées sont identiques, voire plus importants (commandes qui peuvent difficilement attendre et une mutualisation des livraisons forcément plus complexe). Dès lors, on comprend que la diffusion des offres de livraison à domicile en sortie de caisse soit également faible. En effet, pour l'ensemble de l'aire urbaine dijonnaise, seules sept grandes surfaces proposent la livraison à domicile de produits achetés en magasin en dehors de I'hypercentre dijonnais $^{6}$. Ces magasins appartiennent aux enseignes qui proposent aussi un service de vente en ligne dans l'aire urbaine, à savoir Géant Casino, Intermarché et Monoprix. Ainsi, ces enseignes gèrent de manière intégrée le service de livraison à domicile pour leur site de vente en ligne et pour leurs magasins physiques. L'objectif est de parvenir à réaliser des économies d'échelles et à optimiser les moyens mis en œuvre. Intermarché a adopté la forme la plus intégrée : si 6 magasins de l'enseigne (sur les 7 que compte le territoire étudié) offrent la livraison à domicile, un seul service de livraison opère pour l'ensemble de ces magasins et pour la vente en ligne. Ce service est réalisé en propre, sans avoir recours à un prestataire extérieur pour délivrer les produits achetés.

\footnotetext{
${ }^{6}$ Cette zone bénéficie de services de livraison à domicile opérant dans un rayon limité, qui, le plus souvent, ne dépasse pas les limites d'un quartier.
} 
Responsable de la gestion et de l'organisation de livraison pour l'ensemble des Intermarché de la zone, le magasin de Port du Canal propose également le retrait en magasin des commandes effectuées en ligne, même s'il ne dispose pas de "drive " (cf. ante). Si l'organisation de Monoprix est moins complexe (un seul magasin sur l'aire urbaine), l'enseigne a retenu la même stratégie d'intégration qu'Intermarché, à la différence que le service a été externalisé et confié à un prestataire extérieur Cette intégration des différentes fonctions du commerce (en ligne et en magasin) montre l'émergence d'une véritable hybridation (Rallet 2001) ou stratégie multicanale (voire transcanale) de la part de ces enseignes (Moati 2009 ; Poirel et Fernandez 2008).

Au-delà de ces formes intégrées, deux cas plus singuliers émergent dans l'aire urbaine dijonnaise. Le premier est celui de Géant Casino. Le magasin de Chenôve dans le sud de l'agglomération constitue le point de distribution pour le site de vente en ligne de l'enseigne alors que seul le magasin de Fontaine-Lès-Dijon offre la livraison à domicile pour les achats faits en magasin. II semble que le magasin de Chenôve ne souhaite pas offrir de service de livraison à domicile pour les achats effectués en magasin. Le magasin de Fontaine-Lès-Dijon n'a pas nécessairement une volonté plus forte d'offrir ce service qui n'est même pas signalé sur le site web de son enseigne. Pour autant, un prestataire, Maxi'M Services, porte ce service dans le cadre d'un accord déjà ancien. Le magasin n'y est que très peu engagé, voire complètement absent, puisque le logo ou le nom de l'enseigne n'apparaît jamais sur le matériel de promotion (affichettes et prospectus), la camionnette de livraison, les uniformes des livreurs ou les bons de livraison. Pour le magasin, l'intégration de la livraison à la politique commerciale est impensée, au point que le prestataire offre la préparation de commandes passées par mél ou téléphone. II se place ainsi en concurrence directe avec l'offre de vente en ligne de l'enseigne assurée par le magasin de Chenôve. Le second cas, plus original, d'un service de livraison à domicile non intégrée entre vente en magasin et vente en ligne est celui offert par une auto-entrepreneuse en services d'aide à la personne. Elle aide des personnes à faire leurs courses, en les accompagnant ou en allant les faire à leur place. Ayant accompagné plusieurs fois des personnes au magasin Super $U$ de Talant, elle a demandé l'autorisation de pouvoir apposer une affichette en arrière caisse pour proposer ses services liés à la livraison des courses, ce qui lui a été accordé. Elle a indiqué lors de l'entretien qu'elle était susceptible d'offrir ce service dans toutes les grandes surfaces de l'aire urbaine dijonnaise.

En somme, à Dijon et ses alentours, l'offre de livraison à domicile de produits alimentaires à partir des GSA (en magasin ou en ligne) s'avère faiblement développée. Seules quelques enseignes spécialisées dans le "store picking " ont investi ce secteur. Trois d'entres elles ont opté pour une gestion intégrée de la livraison à domicile de leurs produits. Cette option peut être vue comme le résultat d'une politique plus ou moins homogène au sein d'un groupe propriétaire en propre de ces surfaces de vente (Monoprix et Géant Casino Chenôve pour la vente en ligne) ou d'un ensemble de magasins franchisés appartenant aux mêmes propriétaires (Intermarché7). Ces magasins disposent d'une taille suffisamment critique pour proposer un tel service et amortir les coûts associés. Pour Super $U$, les magasins sont possédés par des commerçants propriétaires de leurs magasins et associés au sein d'une coopérative régionale. Dans l'aire urbaine, 10 magasins de l'enseigne sont présents et sont aux mains de 7 propriétaires différents. Présentant des chiffres d'affaires et des

\footnotetext{
${ }^{7}$ Après examen des fichiers SIRET des points de ventes, il apparaît que les 6 magasins Intermarché qui proposent la livraison à domicile sont en fait détenus par le même consortium de propriétaires.
} 
zones de chalandises différentes, il leur est vraisemblablement difficile de proposer un service de manière intégrée. En l'absence d'une politique d'enseigne forte en la matière (Géant Casino Fontaine-Lès-Dijon) ou d'une taille critique nécessaire pour proposer ce service (Super $U$ ), des prestataires extérieurs dûment identifiés proposent la prestation aux consommateurs et sont rémunérés par eux. Les magasins dans lesquels ces prestataires sont les plus visibles sont justement ceux qui ne proposent pas eux-mêmes la livraison à domicile et qui bénéficient donc d'une complémentarité de service sans avoir à investir. Les entretiens et observations de terrain montrent bien que ce partenariat est essentiellement tacite et flou, fruits d'arrangements informels locaux. Dès lors, le service proposé apparaît fragile et sa pérennité est moins garantie.

\section{La livraison à domicile ou un service pour des clientèles précisément ciblées}

Si l'offre de livraison à domicile de l'aire urbaine est limitée, avant de déterminer les périmètres desservis et l'impact de ce service sur l'accessibilité aux produits alimentaires des territoires, il est important de saisir à quelle clientèle tentent de s'adresser les distributeurs. Selon les populations concernées, certains territoires seraient de facto mieux desservis que d'autres. En l'occurrence, la livraison à domicile (à partir de la vente en magasin ou de l'achat en ligne) rentre dans une stratégie servicielle mise en place par les enseignes et s'adresse à plusieurs publics cibles, à savoir les personnes dépendantes et les familles avec enfants. Lorsqu'on les interroge, les acteurs de la livraison à domicile de l'aire urbaine dijonnaise désignent les personnes âgées et/ou à mobilité réduite comme leur cible principale pour les livraisons effectuées à partir des grandes surfaces. Si ces ménages sont parmi les principaux utilisateurs de la livraison à domicile, ils le font plutôt à partir de la vente en magasin, élément important dans leur sociabilité (Barth et Anteblian 2010). Leur part dans la clientèle de la vente en ligne n'a pas pu être déterminée mais elle est vraisemblablement faible, voire inexistante. Cette hypothèse découle des résultats d'autres enquêtes, en France ou à l'étranger, qui mettent en évidence le faible recours des personnes âgées à la vente en ligne (Berret 2008) du fait d'un usage moins important, même si en constante progression, de l'ordinateur et donc d'Internet au sein des tranches d'âges les plus âgées (Bigot et Croutte 2011). Après les personnes âgées, les ménages sans voiture sont les principaux utilisateurs désignés de la livraison à domicile à Dijon, alternative à des approvisionnements fréquents et réguliers ou au transport difficile d'une grande quantité de produits (fragiles, périssables, sensibles au respect de la chaîne du froid, etc.). Ces deux segments (qui peuvent se recouper) sont les cibles privilégiées des prestataires comme celui du Super $U$ de Talant. En effet, ce prestataire propose des services à la personne (dont la livraison des courses mais il peut aussi s'agir d'un accompagnement) plus que du transport de marchandises. Le service proposé relève alors principalement de la prise en charge de la dépendance et du handicap dans le cadre d'un maintien à domicile autonome. Dans le cadre de ce partenariat tacite, Super U Talant est gagnant puisque le service de livraison est proposé dans son magasin sans qu'il ait à le financer. En l'occurrence, les consommateurs et les pouvoirs publics s'en chargent pour lui à travers la prise en charge de la dépendance.

L'autre catégorie principale ciblée est les familles avec enfants qui sont à la fois des utilisateurs privilégiés de la vente en ligne et des cibles équivoques de la livraison à domicile à partir de la vente en magasin car elles achètent, en général, de plus gros volumes que les autres catégories de ménages. Monoprix en fait clairement l'une de ses cibles principales. Pour ce magasin situé en plein cœur du centre-ville de Dijon, une partie importante de ses clients viennent à pieds et ont de petits volumes d'achats. La livraison à domicile a pour objectif d'attirer des familles qui habitent dans le 
centre et se rendent dans les grandes surfaces périphériques pour leurs courses car ces magasins sont plus accessibles en voiture. Elles permettent donc de réaliser des approvisionnements plus conséquents. Le tarif de la livraison à domicile est ainsi dégressif avec le montant d'achat que ce soit pour la vente en ligne ou la vente en magasin afin d'inciter les ménages avec enfants à acheter de plus grandes quantités. D'autres acteurs du marché dijonnais appliquent des politiques tarifaires différentes qui favorisent la livraison en grandes quantités. Ainsi Intermarché a un tarif unique pour la livraison à domicile, quel que soit le montant commandé ou la distance à parcourir, ce qui incite à commander plutôt de grands volumes de marchandises. Pour les prestataires extérieurs présents au Géant Casino de Fontaine-Lès-Dijon et au Super $U$ de Talant, les tarifs de livraison augmentent avec le volume transporté, en particulier pour les packs de boissons. Une journée d'observation participante (suivi de livraisons) avec le prestataire du Géant Casino de Fontaine-Lès-Dijon (vente en magasin) montre que les frais supplémentaires ne sont jamais demandés au client. Les tarifs les plus élevés et/ou non dégressifs de livraison à domicile sont appliqués lorsque les magasins qui offrent ce service n'en sont pas à l'origine et n'en font pas un outil de développement. Cette tendance à favoriser la livraison de grandes quantités en vue de réaliser des économies d'échelle montre que les services de livraisons ciblent les grands ménages et donc les familles avec enfants.

Les horaires de livraisons déterminent également à quelle clientèle les services proposés conviennent le mieux et dont ils sont la cible. Par exemple, pour les magasins Intermarché, les livraisons à domicile peuvent avoir lieu toute la semaine (du lundi au vendredi) jusqu'à $17 \mathrm{~h} 30$ au plus tard. Ces horaires ne permettent pas de viser une clientèle large et ciblent prioritairement les personnes âgées ou inactives. Pour les ménages biactifs, notamment avec enfants, ces horaires sont moins compatibles avec leurs propres contraintes temporelles (temps professionnels et scolaires). Pour cette raison, Monoprix et Géant Casino ont des plages de livraison qui s'étendent jusqu'à 21h en semaine et qui ont lieu le samedi. Si les horaires sont plus étendus, certaines enseignes qui proposent la vente en ligne tendent aussi à favoriser la promotion du retrait en magasin ou en drive auprès de ce type de public. En effet, pour Intermarché (retrait en magasin) et Géant Casino (drive), les courses commandées sont récupérables tous les jours, de $9 \mathrm{~h}$ à $19 \mathrm{~h}$ ou $20 \mathrm{~h}$, soit sur des plages beaucoup plus étendues que la livraison à domicile. Les enseignes jouent sur deux tableaux complémentaires. Avec la vente en ligne, ils proposent aux ménages biactifs une alternative à la pénibilité associée à des achats corvée (de Coninck 2010), plus facilement substituables par des courses faites en lignes. Mais, le manque de disponibilité des ménages biactifs rend plus difficile, du point de vue logistique, l'organisation d'une livraison à domicile pour des volumes importants comme les courses hebdomadaires et plus particulièrement dans les espaces moins denses comme le périurbain. En effet, cela suppose d'effectuer les livraisons sur des créneaux courts ou à des heures exactes et en dehors des horaires standards de travail, à savoir le soir ou/et les week-ends. Or, une telle organisation logistique s'avère très couteuse pour les distributeurs contrairement à des livraisons avec des fenêtres temporelles plus larges ou sans créneaux horaires (Gevaers et al. 2011). Outre la mauvaise perception d'une livraison inattendue, elle est d'autant plus difficile à mettre en place pour le distributeur qu'elle suppose un respect de la chaîne du froid et la mise en place de dispositifs comme des consignes à compartiment réfrigéré qui sont coûteuses. Une autre stratégie émerge pour réduire au maximum la prise en charge du dernier kilomètre. En jouant sur les pratiques des consommateurs en quête permanente d'instantanéité, les distributeurs alimentaires tendent à favoriser des modes de distribution hors domicile comme le " drive " (Gasnier 2007). Ils évitent ainsi de nombreux coûts inhérents à la desserte de territoires peu denses (Boyer et al. 2009; 
Punakivi et Saranen 2001) en argumentant sur le coût moindre du système pour le consommateur qui trouve toujours trop élevés les frais de livraison liés à l'achat en ligne en général et à l'épicerie en ligne en particulier ${ }^{8}$ (Bitoun 2009). Ce faisant, pour Intermarché et Géant Casino, avec le " drive », les consommateurs restent en charge du dernier kilomètre.

Au final, pour les magasins qui proposent des services de livraison à domicile, les cibles visées sont avant tout les personnes à mobilité réduite, dépendantes d'autrui pour réaliser leurs courses et qui préfèrent a priori se rendre en magasin puis se faire livrer. Leur seconde cible, les familles avec enfants, n'a pas forcément une stratégie unique et peut alterner entre achats en magasin et achats en ligne, livraison à domicile et récupération des courses en drive. Dans les deux cas, les populations ciblées sont présentes dans l'ensemble du territoire investigué. Les familles avec enfants représentent un idéal-type des ménages périurbains et les personnes âgées y sont constante augmentation en vertu du vieillissement de ces espaces (Berger et al. 2010). Outre la faiblesse de l'offre proposée, malgré une clientèle diversifiée et présente dans tous les types de territoires, les enseignes tendent à desservir de manière inégale les différents territoires de l'aire urbaine dijonnaise.

\section{Le périmètre desservi : du bricolage à l'évitement des territoires les moins denses}

En observant dans un premier temps les territoires desservis par les cybermarchés, la somme de leurs périmètres de livraison (cf. carte $n^{\circ} 1$ ) ne permet de couvrir que $25 \%$ de la surface de l'aire urbaine, mais touche plus de $80 \%$ de sa population. Le périmètre le plus étendu est celui de "MesCoursesCasino " qui englobe $77 \%$ de la population pour seulement $20 \%$ de la surface, et le plus réduit est celui de Monoprix avec $70 \%$ de la population et $14 \%$ de la surface. Dès lors, les périmètres de livraison des cybermarchés se chevauchent fortement. En effet, ils englobent toutes les communes du pôle urbain, c'est-à-dire les communes les plus peuplées. Outre leur niveau de peuplement, le choix de ces communes est aussi lié au fait que les périmètres retenus sont centrés autour de leurs points de distribution respectifs, tous situés à Dijon ou dans une commune limitrophe. Ce choix logistique vise à minimiser les distances à parcourir pour livrer les clients comme en témoignent les ajustements effectués par Télémarket (devenu depuis UTelemarket) en Île-deFrance. A la fin des années 1990, son premier entrepôt logistique était situé à Rungis alors que l'essentiel de sa clientèle se situait dans Paris, $15 \mathrm{~km}$ plus loin. Cette localisation s'est rapidement avérée trop éloignée. Ainsi, entre 1999 et 2001, l'entrepôt a changé deux fois de localisation pour se rapprocher progressivement de Paris (Colin 2001).

Par conséquent, les zones périphériques de l'aire urbaine restent hors de la zone de livraison car elles sont trop éloignées du point de distribution et comprennent peu de clients potentiels. Pour certains e-marchands qui promettaient la desserte de territoires ruraux, l'argument publicitaire s'avère mensonger puisque, contrairement à son slogan, l'enseigne dessert seulement six communes de I'aire urbaine dijonnaise et aucune d'entre elles n'est périurbaine. Si les autres enseignes proposent des aires de livraison plus étendues, la promesse d'un accès sans contrainte de transport à une offre de produits de supermarchés reste impossible dans la plupart des communes de l'aire urbaine

\footnotetext{
${ }^{8}$ Peu importe la nature du produit acheté.
} 
dijonnaise. De plus, la livraison à domicile par les cybermarchés tend à renforcer les inégalités d'accès au commerce des communes périurbaines par rapport aux communes du pôle urbain. Ce dernier bénéficie déjà des principales implantations des grandes surfaces alimentaires du centre et des principales zones commerciales d'entrée de ville. Désormais, il profite aussi de la livraison à domicile des cybermarchés. Pour autant, un certain nombre de communes de la première couronne périurbaine sont incluses dans le périmètre de la livraison à domicile, ce qui améliore leur accès aux produits alimentaires, en particulier pour les populations les moins mobiles. Pour les communes de la seconde couronne périurbaine dijonnaise, l'inclusion dans un périmètre de livraison à domicile pourrait avoir lieu si le point de distribution était basé dans une grande surface d'une zone commerciale périphérique plutôt que dans une grande surface du centre. Ainsi, à défaut d'un magasin dans le centre, Géant Casino assure la distribution des ventes de son cybermarché à partir de son magasin de Chenôve au sud du pôle urbain. Cela lui permet d'inclure de nombreuses communes du sud de l'aire urbaine dans son périmètre de livraison, mais renchérit probablement d'autant le coût des livraisons dans la commune de Dijon et dans la partie nord du pôle urbain. Toutefois, on peut douter que le marché dijonnais soit suffisamment important pour permettre une organisation par secteur de la livraison à domicile des cybermarchés. 


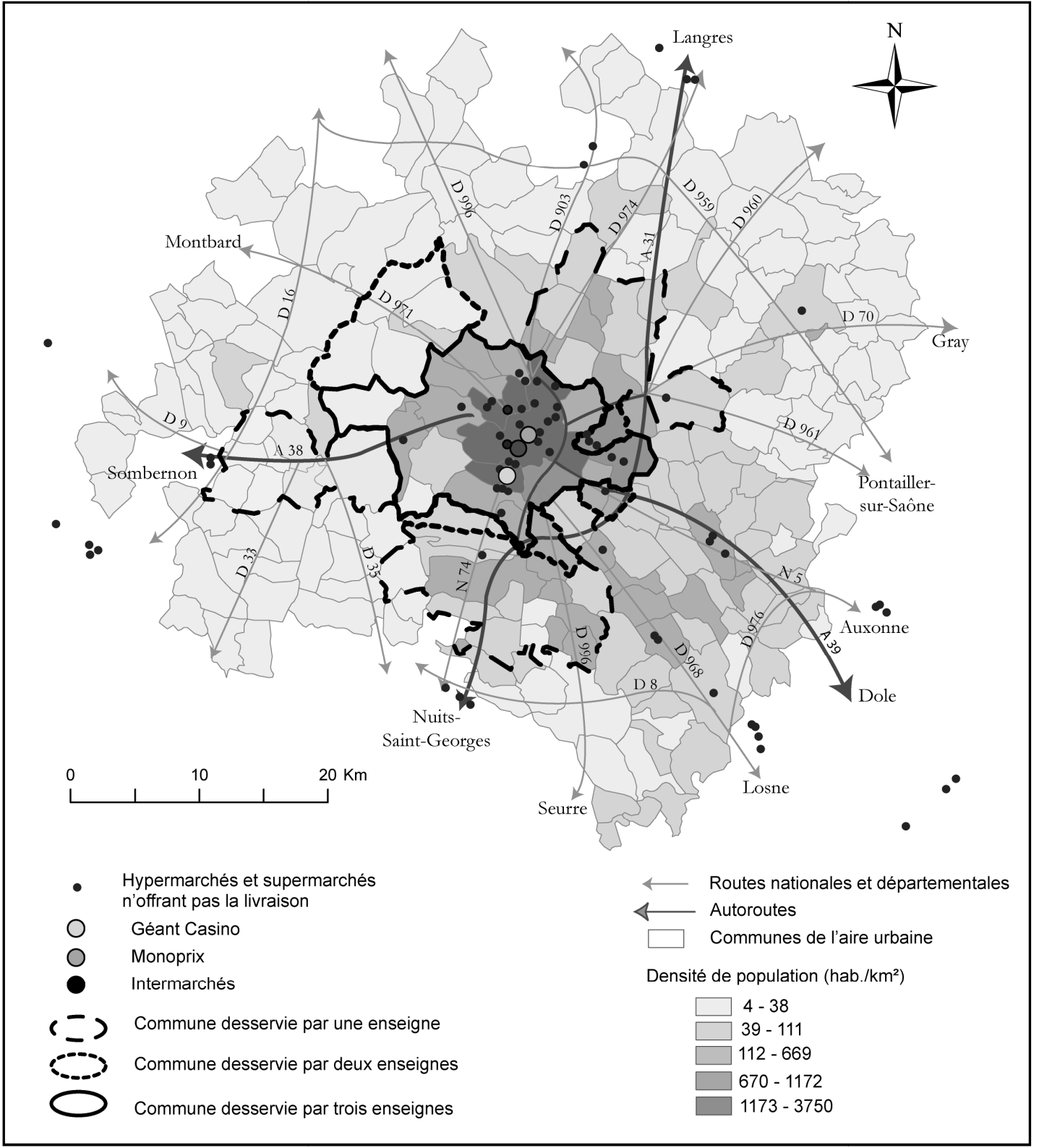

Réalisation et source : Laboratoire ThéMA 


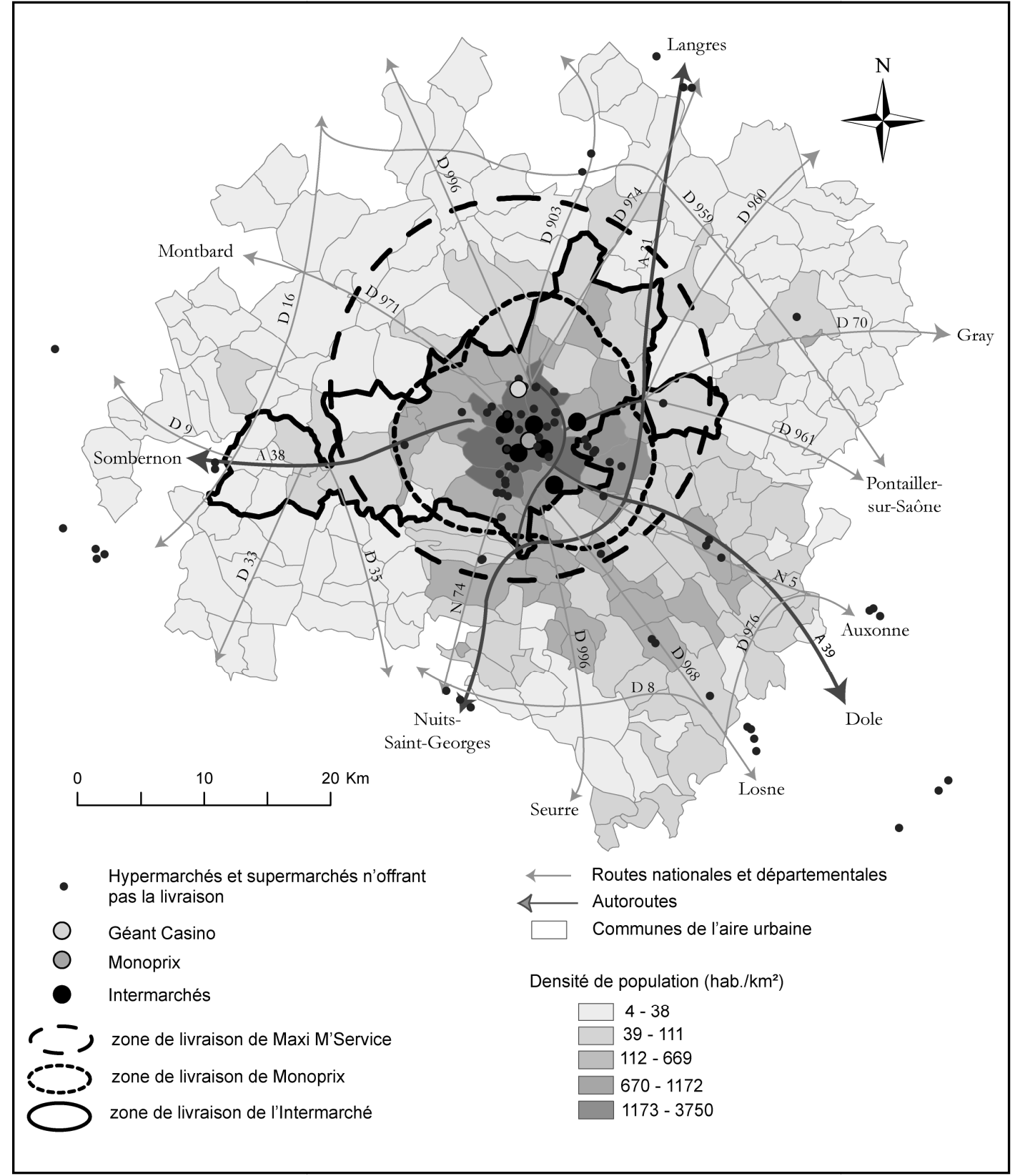

Réalisation et source : Laboratoire ThéMA

Au-delà d'un schéma concentrique autour des points de distribution, des excroissances se dessinent dans les périmètres de livraison. Elles correspondent tout d'abord à des zones de forte densité. Ensuite, les zones de livraison s'étirent le long des principaux axes routiers radiaux, par exemple dans l'ouest de l'aire urbaine le long de l'autoroute A38, où Intermarché livre jusqu'à 23 kilomètres de son point de distribution. Enfin, les codes postaux modèlent également les périmètres de la livraison: pour des questions de facilité d'appréciation par la clientèle de leurs zones de livraison, Monoprix et Casino utilisent les codes postaux qui indiquent au client si une commune peut être livrée ou non. Ce choix a pour effet d'ajouter à leurs périmètres de livraison des groupes de communes dont certaines sont relativement éloignées du point de distribution et affichent de très faibles densités. Par exemple, dans le secteur nord ouest de Dijon encadrant la route D971, le code 21121 correspond à 
sept communes différentes (Ahuy, Daix, Darois, Etaule, Fontaine-Lès-Dijon, Hauteville-Lès-Dijon et Val-Suzon). Si certaines communes sont limitrophes ou à proximité immédiate de Dijon (Ahuy, Daix, Fontaine-Lès-Dijon et Hauteville-Lès-Dijon), celle de Val Suzon (la plus éloignée de Dijon), à son extrémité nord-ouest, est située à 17 kilomètres par la route du centre de Dijon et du magasin Monoprix et à plus de 20 kilomètres du magasin Géant Casino de Chenôve.

Les périmètres de livraison à domicile sont également marqués par l'absence d'extension à l'est du pôle urbain et particulièrement le sud-est dans la plaine de la Saône. Cette partie du périurbain dijonnais affiche pourtant de plus hauts niveaux de densité car elle comporte de petites unités urbaines, telles que Genlis et ses 15000 habitants située à 20 kilomètres de Dijon et bien reliée par la route. L'absence de ce secteur dans les périmètres de livraison à domicile peut s'expliquer par la présence de plusieurs grandes surfaces dans cette zone. Ainsi les cybermarchés en dehors du cœur de l'agglomération chercheraient à éviter la concurrence des grandes surfaces locales. Un autre facteur pourrait être le plus faible niveau de revenu dans cette partie de l'aire urbaine, en particulier par rapport à sa partie ouest. Le périmètre de livraison du cybermarché d'Intermarché englobe les communes qui connaissent une forte concentration de cadres à l'ouest et au nord de Dijon. Les extensions du périmètre de livraison à domicile le long de l'A38 à l'ouest et entre la D974 et l'A31 au nord relèvent de cette logique (voir carte 1). Le périmètre de la livraison à domicile d'Intermarché offre aux cadres une réduction de la distance d'accès plus importante que pour les autres catégories (7). Ce n'est pas le cas des périmètres de livraison à domicile des cybermarchés de Casino et Monoprix qui ne semblent pas plus favoriser les cadres que les autres catégories de ménages. Alors que le positionnement plus haut de gamme de ces deux enseignes, et en particulier de Monoprix, par rapport à Intermarché, aurait dû les inciter à favoriser en premier lieu les cadres.

Pour les achats effectués en magasin, les aires de livraison (cf. carte $n^{\circ} 2$ ) sont plus petites que celles de la vente en ligne, à l'exception de celle d'Intermarché qui est identique. Les livraisons offertes en sortie de caisse sont, par nature, plus imprévisibles et plus urgentes. Elles doivent être traitées dans I'heure alors que les livraisons pour la vente en ligne sont programmées au moins vingt-quatre heures à l'avance. De ce fait, les aires de livraison tendent à être plus restreintes pour disposer de marges de manœuvre en cas de surcroît d'activité ou de destinations trop divergentes. L'autre caractéristique forte des aires de livraison pour les achats effectués en magasin est leur délimitation floue. Que ce soit pour Monoprix ou pour Géant Casino, la seule indication donnée concerne un rayon d'une quinzaine de kilomètres autour du magasin sans savoir s'il s'agit d'une distance euclidienne ou d'une distance réseau. Interrogé sur la desserte d'une commune à la limite euclidienne des quinze kilomètres, le prestataire a une réponse plutôt affirmative, mais peu ferme, relative au retour d'expérience d'une première livraison. II se réserve la possibilité de refuser une autre livraison s'il estime avec l'expérience que cette destination est finalement trop éloignée. Dans les faits, l'aire de desserte définie par un rayon autour du magasin fait partie de l'accord avec le magasin et doit être théoriquement opposable au prestataire. Mais le mode de calcul de la distance y est-il précisé ? Par ailleurs, pour tous les livreurs, les demandes sont concentrées autour du magasin et les situations potentiellement litigieuses semblent rares, voire inexistantes.

Pour conclure, toutes enseignes confondues, le périmètre de la livraison à domicile d'achats alimentaires (en magasin ou en ligne) reste limité et centré autour de Dijon : il s'étend peu au-delà de l'agglomération (cf. cartes $n^{\circ} 1$ et 2 ). Les territoires périurbains et les communes peu dotées en 
grandes surfaces alimentaires sont celles les moins desservies par la livraison à domicile. De ce fait, les inégalités territoriales en termes d'accessibilité aux commerces se voient renforcées.

\section{CONCLUSION}

Au final, l'étude de la livraison à domicile, de son organisation et des périmètres desservis sur l'aire dijonnaise remet en cause la possibilité d'une accessibilité plus homogène des produits alimentaires d'un territoire à l'autre. Les distributeurs tendent à favoriser les territoires les plus denses et les plus peuplés pour maximiser leur part de marché et réduire au maximum les coûts liés à la desserte du dernier kilomètre. A l'heure actuelle, nous serions plutôt face au renforcement de zones au détriment des territoires périurbains peu denses ou ruraux plutôt qu'à un maillage plus homogène des territoires d'une aire urbaine (Rallet 2001). En termes d'usages d'Internet, ce constat va à l'encontre d'un lien positif entre technologies de l'information et de la communication et étalement urbain.

Plusieurs arguments tendent à supposer que cet état de fait n'est pas encore figé même s'il n'est pas sûr que l'accessibilité aux produits alimentaires des territoires dépendants de l'automobile s'améliore. A l'exemple de l'aire urbaine dijonnaise, d'une enseigne à l'autre, le service proposé n'est pas identique. Les zones desservies varient fortement selon la politique de l'enseigne en la matière et la capacité de cette dernière à la faire appliquer, notamment en fonction de la structure juridique des magasins (magasin franchisé isolé, magasins franchisés aux mains du même propriétaire, magasins possédés en propre). Pour les cybermarchés ou les magasins, l'offre de livraison à domicile évolue parfois brusquement et les périmètres de livraison tels qu'ils existent aujourd'hui ne sont pas nécessairement durables. En plus des accords locaux et tacites conclus par des magasins avec des prestataires extérieurs, les cybermarchés ne constituent pas forcément une offre stabilisée. Ainsi, ils peuvent disparaître à l'image du premier site de vente en ligne créé par Casino qui s'est relancé quelques années plus tard sur le créneau des cybermarchés (Durand 2010). Les périmètres de livraison peuvent donc radicalement changer et voir leur taille évoluer à l'image d'Houra dont le périmètre a été réduit ou de UTelemarket dont le sien n'a jamais augmenté malgré plusieurs annonces en ce sens (Gavaud 2010, Durand et Senkel 2007). Contrairement à d'autres types de produits (produits culturels), l'offre de produits alimentaires proposée principalement par les cybermarchés progresse peu. Le chiffre d'affaires des cybermarchés augmente lentement et, à ce jour, aucun des cybermarchés ne s'est révélé durablement rentable (Ranvier et Sury 2009). L'offre existante reste donc fragile et la livraison à domicile, qui représente l'un des postes de dépense les plus élevés, n'a pas encore trouvé son modèle en termes d'organisation ou d'étendue de son périmètre en dehors des zones les plus denses. Cette absence de modèle est par ailleurs entretenue par l'émergence des " drive " ou des modes de retraits en magasins qui permettent aux enseignes de renoncer à ce poste de dépenses. Des ajustements sont probables dans les communes en marge des périmètres, tandis que celles situées dans l'espace central peuvent avoir l'assurance d'un service continu et du choix entre plusieurs enseignes. Au final, à moins d'une explosion de la demande des cybermarchés ou de la livraison à domicile à partir de l'achat en magasin, les inégalités de desserte des domiciles se renforceront si le service est laissé aux seules mains des distributeurs. 
Néanmoins plusieurs arguments laissent supposer que les pouvoirs publics ont un rôle potentiel à jouer si on suppose que les choix de localisation des ménages évolueront peu. En effet, là où le service n'est pas proposé (à partir de la vente en magasin comme de la vente en ligne), des initiatives individuelles tendent à se substituer aux distributeurs. Transporteurs, services à la personne rendent possible ce service en l'économisant aux magasins. De ce fait, clients et pouvoirs publics assument le coût financier de la livraison à domicile. En effet, dans le cadre des dispositifs de prise en charge de la dépendance et la promotion des services à la personne, les Conseils Généraux (qui financent l'allocation personnalisée d'autonomie et donc la prise en charge de la dépendance des personnes âgées et/ou handicapées) et le trésor public (crédit d'impôts pour les autres ménages) participent plus ou moins directement à la prise en charge du service. D'autres modalités de desserte des territoires peuvent être imaginées comme l'installation de consignes réfrigérées individuelles ou collectives pour permettre des livraisons non attendues, un partenariat avec un commerce de détail local, etc. Ces modalités interrogent cependant sur leur prise en charge financière et le rôle des pouvoirs publics locaux dans leurs mises en place. A quel point pourront-ils ou voudront-ils participer à l'émergence de telles solutions qui sont encore coûteuses et qui peuvent être prises en charge par d'autres acteurs?

\section{BIBLIOGRAPHIE}

Augereau V., Currien R. et Dablanc L., 2009, « Les relais-livraison dans la logistique du e-commerce, l'émergence de deux modèles ». Cahiers scientifiques du transport, Vol.55, pp.63-95.

Barth I., Anteblian B., 2010, "Seniors, grande distribution et courses ordinaires ». Gérontologie et société, Vol.135(4), pp.83-113.

Berger M., Rougé L., Thomann S. et Thouzellier C., 2010, "Vieillir en pavillon : mobilités et ancrages des personnes âgées dans les espaces périurbains d'aires métropolitaines (Toulouse, Paris, Marseille) ». Espace populations sociétés, Vol. 2010/1, pp.53-67.

Berret P., 2008, «Diffusion et utilisation des Tic en France et en Europe ». Culture chiffres, Vol.2(2), pp.1-15.

Bigot R., Croutte P., 2008, La diffusion des technologies de l'information et de la communication dans la société française, Paris : CREDOC, Rapport.

Bitoun O., 2009, E-commerce et distribution : comment Internet bouscule les canaux de vente, Paris : Acsel.

Boyer K.K., Prud'homme A.M. et Chung W., 2009, " The last Mile Challenge: evaluating the effects of customer density and delivery window patterns ». Journal of Business Logistics, Vol.30(1), pp.185201.

Bras M.-A., Pégaz-Blanc O. et INSEE, 2012, Tableaux de l'économie française, Edition 2012, Paris : INSEE.

Brousseau E., Kessous E., 2003, Impacts des NTIC sur les modèles logistiques du commerce électronique : nouveaux métiers, nouvelles formes d'intermédiation ?, Paris : PREDIT, Rapport. 
Cairncross F., 1997, The Death of Distance: How the Communications Revolution Will Change Our Lives. Harvard Business Press.

Cairns S., 2005, « Delivering supermarket shopping: more or less traffic? ». Transport Reviews, Vol.25 (1, pp.51-84.

Colin J., 2001, "The impact of e-commerce on logistics ", The Impact of E-commerce on Transport, Paris.

Cullinane S., 2009, «From Bricks to Clicks: The Impact of Online Retailing on Transport and the Environment ». Transport Reviews, Vol.29(6), pp.759-776.

De Coninck F., 2010, "L'achat en ligne, un nouveau rapport à l'espace de la consommation ». Sociologies pratiques, Vol.20(1), pp.51-62.

Dumans M.-E., Chambolle C., 2002, "Internet et la grande distribution alimentaire française ». Économie rurale, Vol.272(1), pp.42-56.

Durand B., 2010, "e-commerce et logistique urbaine: quand le développement durable $s^{\prime}$ en mêle... ». Revue Française de Gestion Industrielle, Vol.29(2), pp.7-26.

Durand B. 2008, « Les magasins de proximité : un atout logistique pour l'épicerie en ligne ». La Revue des Sciences de Gestion, Vol.229(1), pp.75-83.

Durand B., Senkel M.-P., 2007, «La logistique de l'épicerie en ligne: vers une différenciation des solutions ». Décisions Marketing, n45, pp.75-89.

FEVAD, 2011, Chiffres clés, Vente à distance e-commerce aux particuliers, Paris : FEVAD.

Gasnier A., 2007, "Les temps de mobilité des consommateurs au cœur des nouvelles logiques d'implantation de l'offre commerciale ». Espace populations sociétés, Vol.2007/2-3, pp.243-254.

Gavaud O., 2010, Les déplacements liés aux nouveaux modes d'achat des produits de consommation courante. Approche comparative de l'efficience écologique de l'achat classique en magasin, du ecommerce, du drive-in et de la livraison de caddies, Nantes: CETE de l'Ouest, Rapport.

Gevaers R., Van de Voorde E. et Vanelslander T., 2011, "Charateristics and Typology of Last-mile Logistic from an Innovation Perspective in an Urban Context ». In C. Macharis et S. Melo (eds), City Distribution and Urban Freight Transport: Multiple Perspectives, pp.56-71. Edward Elgar Publishing.

Gratadour J.-R., 2001, « La logistique du commerce électronique ». Réseaux, 106(2), pp.135-147.

INSEE, 2010. Le commerce en France édition 2010, Paris: INSEE.

Koster R., 2002, "Distribution structures for food home shopping ». International Journal of Physical Distribution \& Logistics Management, Vol.32(5), pp.362-380.

Li F., Yousept I., 2004, "Online Supermarkets: Emerging Strategies And Business Models In The UK ». 17th Bled eCommerce Conference, Bled (Slovenia). 
Moati P., 2009, La Vente à distance dans la nouvelle révolution commerciale, Paris : CREDOC, Rapport.

Motte-Baumvol B., 2008, "L'accès des ménages aux services dans l'espace périurbain francilien ». Strates, Vol.14, pp.149-164.

Murphy A.J., 2007, "Grounding the virtual: The material effects of electronic grocery shopping ". Geoforum, Vol.38, No.5, pp.941-953.

Ogawara S., Chen J.C.H. et Zhang Q., 2003, "Internet grocery business in Japan: current business models and future trends". Industrial Management \& Data Systems, Vol.103(9), pp.727-735.

Poirel C., Fernandez D.B., 2008, "La stratégie de distribution multiple. À la recherche de synergies entre canal physique et canal virtuel ». Revue française de gestion, Vol.182, No.2, pp.155-170.

Punakivi M., Saranen J., 2001, "Identifying the success factors in e-grocery home delivery ". International Journal of Retail \& Distribution Management, Vol.29, No.4, pp.156-163.

Rallet A., 2001, "Commerce électronique et localisation urbaine des activités commerciales ». Revue économique, Vol.52(7), pp.267-288.

Ranvier M., Sury R., 2009, La vente de produits alimentaires sur Internet : un état des lieux en 2009. Paris : CREDOC, Rapport.

Shen Q., 2000, "New telecommunications and residential location flexibility ". Environment and Planning A, Vol.32(8), pp.1445-1463. 